\title{
CORRESPONDENCE
}

\section{Reply to 'In vivo imaging of islet transplantation'}

\section{To the editor:}

In vivo visualization of transplanted pancreatic islets might advance islet transplantation as treatment for diabetes, helping to confirm technical success and diagnose rejection. Evgenov et al. ${ }^{1}$ described a method of labeling human islets with a superparamagnetic contrast agent and the subsequent detection of the islets using magnetic resonance imaging after transplantation into the liver or the renal subcapsular space in mice. The islets or islet clusters were apparent as hypointense spots on T2-weighted magnetic resonance images for up to 180 days.

Although we appreciate the technical excellence and possible application of this method in clinical practice, we disagree with the interpretation of our previous report offering very similar data using the clinically approved superparamagnetic magnetic resonance contrast agent ferucarbotran in a rat model. We clearly showed that transplantation of islets in vitro-labeled with iron successfully treated experimental diabetes and that the presence of iron-labeled islets transplanted into the liver could be successfully monitored on serial magnetic resonance scans for as long as 22 weeks after transplantation ${ }^{2}$.

In addition, we attempted to estimate the average iron content in islets following a 2day culture in the presence of ferucarbotran. We object to the characterization of our result as "seem[ing] unrealistic" as a value for iron uptake per cell, as we had not reported uptake by single cells. The amount of iron per cell was calculated from the iron measured in islet samples. Soon after placement in the culture medium, iron within an islet is localized not only to intracellular but also to extracellular spaces, which we stated clearly ${ }^{2}$.

We were also surprised that Evgenov et al. ${ }^{1}$ referred to our paper only regarding the quantification of islet iron content and the impact of ferucarbotran on in vitro islet function. We were the first to report on the use of magnetic resonance imaging for in vivo islet visualization, and this fact was not included in their article. Although ours was only a pilot study, our results clearly showed that in vivo imaging of transplanted islets is feasible. As ferucarbotran labeling did not substantially impair in vitro and in vivo islet function, we believe that our technique of islet visualization using this agent is closer to application in human islet transplantation.

COMPETING INTERESTS STATEMENT The authors declare no competing interests.

Frantisek Saudek, Peter Girman, Jan Kriz, Daniel Jirak \& Milan Hajek

Institute for Clinical and Experimental Medicine, Videnska 1958/9, 14621 Praha 4, Czech Republic. e-mail:frantisek.saudek@medicon.cz

1. Evgenov, N.V., Medarova, Z., Dai, G., Bonner-Weir, S \& Moore A. Nat. Med. 12, 144-148 (2006).

2. Jirak, D. et al. Magn. Reson. Med. 52, 1228-2333 (2004)

\section{Evgenov et al. reply:}

Saudek et al. ${ }^{1}$ express two major concerns regarding our analysis of their results. First, they object to our interpretation of their reported cellular iron uptake. We would like to point out that we did not interpret, but only reiterated their own statement that the reported amount of iron was unrealistically high: "If we assume that one islet is composed of roughly 3000 cells, this may represent approximately $90 \mathrm{pg}$ of iron per cell. This amount is, however, much higher than that found in other cell types"1. We do not believe that it is reasonable for Saudek et al. to criticize us for simply repeating a statement from their own manuscript. Indeed, the authors provide some justification for this observation: "...some iron could remain trapped inside the islet, that is, did not enter the cells but was not washed out." Although we do not object to this possibility, we believe that, until proven experimentally, it remains speculative.

Second, Saudek et al. express disappointment that we did not acknowledge their primacy on the use of magnetic resonance imaging for islet visualization. Fair scientific discourse is founded upon the responsibility for citing the work of others, both as a means of giving credit and as part of an open research dialogue. In agreement with this principle, we expressed our concerns regarding the statements and conclusions made in Jirak et al. ${ }^{1}$ Although we are not disputing the fact that their manuscript came out first, we would like to emphasize that, in our opinion, their publication has important shortcomings. T2 relaxation times in the hundreds of milliseconds showing no correlation with the number of labeled islets, different pulse sequences for experimental and control images, the unknown nature of the causes behind the reported detrimental drop in insulin secretion, and the absence of basic histological studies supporting the imaging data are a few of them. Consequently, we were reluctant to discuss this study in detail and focused only on some results relevant to our manuscript ${ }^{2}$.

Noninvasive imaging of transplanted pancreatic islets has the potential to advance the successful implementation of clinical islet transplantation for diabetes treatment. Hence, the translation of this technique into clinical trials imposes a tremendous responsibility on researchers and requires a thorough and reliable investigation of all possible mechanisms behind islet labeling and all the consequences that it might have on patient health.

\section{COMPETING INTERESTS STATEMENT}

The authors declare no competing financial interests.

Anna Moore ${ }^{1}$, Zdravka Medarova $^{1}$, Natalia $V$ Evgenov $^{1}$, Guangping Dai ${ }^{1} \&$ Susan Bonner-Weir ${ }^{2}$

${ }^{1}$ Athinoula A. Martinos Center for Biomedical Imaging, Department of Radiology, Massachusetts General Hospital, Building $149,13^{\text {th }}$ Street, Room 2301, Charlestown, Massachusetts 02129, USA. ${ }^{2}$ Joslin Diabetes Center, Harvard Medical School, One Joslin Place, Boston, Massachusetts 02215, USA. e-mail:amoore@helix.mgh.harvard.edn

1. Jirak, D. et al. Magn. Reson. Med. 52, 1228-1233 (2004).

2. Evgenov, N. V., Medarova, Z., Dai, G., Bonner-Weir, S. \& Moore, A. Nat. Med. 12, 144-148 (2006) 